\title{
A Building Reconstruction Method Integrated with LiDAR and DLG
}

\author{
Jia Zongren \\ School of Land Science \& Technology \\ China University of Geosciences \\ Beijing, China \\ jiajia_oyeah@163.com \\ Sun Yi-bo \\ School of Land Science \& Technology \\ China University of Geosciences \\ Beijing, China \\ 776746716@qq.com
}

\author{
Zheng Xinqi \\ School of Land Science \& Technology \\ China University of Geosciences \\ Beijing, China \\ zxqsd@126.com \\ Ai Gang \\ School of Land Science \& Technology \\ China University of Geosciences \\ Beijing, China \\ 81693993@qq.com
}

\begin{abstract}
Using airborne light detection and ranging (LiDAR) point cloud data for building reconstruction, is widely used in urban construction, planning and dynamic monitoring, etc. So far, it is still difficult to applied in rapid modeling in large areas. In this study, based on the GIS platform, with the utilization of large scale digital line graph (DLG) combined with airborne LiDAR point cloud data, a rapid modeling method is established. The basic idea is to segment point cloud through histogram threshold analysis and DTM test, then to extract elevation from point cloud by the use of DLG, finally to build the 3D model. In study area, by comparing the different density of the point cloud, result shows that the combination of DLG and airborne LiDAR point cloud, can effectively increase the speed of extracting building elevation information in a large area, so as to achieve the aim of rapid modeling. This approach requires lower density of points than the previous approaches. This study can be widely applied in urban planning, intensive land-use and other aspects.
\end{abstract}

Keywords-point cloud, building reconstruction, histogram threshold, Kriging interpolation

\section{INTRODUCTION}

Light detection and ranging(LiDAR) is an active detection technology that appears recently. It's able to obtain locations and elevation of earth's surface accurately, providing a new means of building reconstruction. Airborne LiDAR system uses helicopter, airship, fixed wing aircraft as carrier, integrated inertial measurement unit, dynamic differential GPS(DGPS) and digital camera unit, and it can collect a large area of spatial data. After software processing, airborne LiDAR point cloud can generate high precision digital terrain model(DTM), digital elevation model(DEM) etc.

In previous study, from the view of data source, research methods are mainly divided into two categories: one is using LiDAR data only, the other is combination of LiDAR data and other remote sensing image. Method of only using point cloud to reconstruct building are widely applied abroad, including based on data's geometric attribute[1-2], LiDAR data classification based on super-voxels and multi-scale[3], specific segmentation rules based on high quality dependence[4], based on Fourier transform[5] etc. These methods all have in common that they are based on traditional analysis approaches of remote sensing images, using algorithms and rules, considering the attributes and characteristics of the data itself segmentation of point cloud data. In short, these methods are suitable for flat terrain and simple shape buildings of large area. The shortcomings are uncertainty heavy computation, and they require high density of LiDAR data.

Compared with only using point cloud for modeling, combining LiDAR data with other remote sensing images to reconstruct buildings can reduce uncertainty and the amount of calculation. It's able to apply to complex building reconstruction of large region. Due to the diversity of the remote sensing images, the domestic and foreign research methods become more abundant. For instance, aerial image[6] and $2 \mathrm{D}$ vector diagram and video image sequence[7] are in use, Split-Merge-Shape[6], Coarse-to-Fine[7], self-adaptable Canny edge detection algorithm[8] are applied in the building reconstruction. These approaches are not affected by topography ups and downs, but the modeling velocity is slow.

This paper belongs to the second kind of methods. The main goal is to establish a point cloud filtering method based on the GIS platform. The main purpose of selecting DLG as data source is its substantivity and accurate outline of building. The structure of this paper is as follow: Chapter 2 introduces the technological method of this study in detail; Chapter 3 presents the experimental results; Chapter 4 is the result evaluation; In Chapter 5, the study conclusion will be stated.

\section{METHOD}

The technical process of this study is divided into 2 steps: (1) obtain the elevation of buildings. (2) building reconstruction. In Step 1, we filter point cloud by histogram 
threshold, then Kriging interpolation is applied to generate DTM to test building points. Through examining the results, we adjust the threshold interval to improve filtering accuracy. Based on GIS platform, overlay analysis of building points and DLG is also considered as a filter process, which eliminates some points out of building contour. After intersect analysis, we extract building elevation information from the DLG. In Step 2, we combine building outline provided by DLG, building elevation in Step 1 and DTM in Step 1 as base elevation to reconstruct buildings. Figure 1 is the specific technique process.

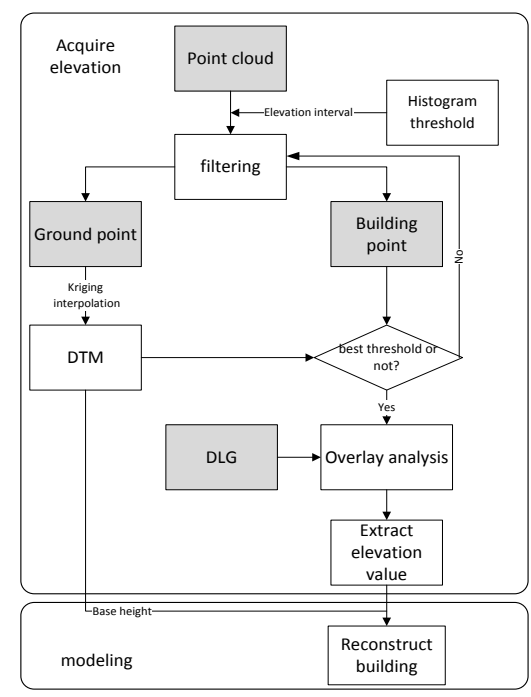

Fig.1. The specific technique process

\section{A. Histogram Threshold Analysis}

Histogram threshold segmentation is usually applied in remote sensing imagery segmentation, as its simple implementation and small calculation quantity. An image is divided into non-overlapping regions by its property as gray level, color and texture[8]. The basic concept of histogram threshold is that a series of data are separated into 2 categories by the best threshold, and the data attribute present two irrelevant subintervals.

The reason why we introduce histogram threshold in this research is that the elevation of point cloud is a continuous interval. Because of the significant difference elevation between ground points and building points, we can segment point cloud by choosing the right elevation statistical intervals and mergering points with the similar elevation. In general, although there are many buildings in urban area, the amount of ground points are still larger than the number of building points. In addition, the elevations of ground points undulate small and present a maximum peak in elevation-frequency histogram. The elevation of two wave trough near peak is the extreme point of ground point elevation interval $\mathrm{H}$. All points within the interval $\mathrm{H}$ can be regarded as the ground points. Obviously the points of which are less than the ground point altitude range is not the building points, so we see them as ground points. The points can be consider as building points of which elevation are greater than the ground point elevation. In histogram threshold segmentation, it's very important to select appropriate statistical interval of elevation. If the gap is too large, data segmentation will be fuzzy. On the contrary, If the gap is too small, it can be hard to distinguish extremal points of frequency shift. Histogram threshold segmentation consists of 3 steps: (1) establish the frequency-elevation histogram, and select appropriate elevation statistics interval. (2) confirm the positions of peak and extremal points. (3) merge intervals.

\section{B. Kriging Interpolation Test}

Kriging interpolation is also called spatial autocovariance optimal interpolation method. This approach not only considers the relative position of observation points and estimative points, but also relative position relationship between observation points. It is a spatial estimation of linear, unbiased, minimum variance. The final goal of this approach is solving the weight coefficient of each fitting points and interpolating the attribute value according to data trend.

In this paper, we choose ground points for interpolation and get the DTM of terrain in study area. DTM aims to test the coincidence between building points after histogram threshold segmentation and elevation trend of DTM. As a reference, we remove part of gross error points which deviate from the trend. If there are many gross error points in test, it means it's not the best threshold in segmentation. Return to threshold analysis to choose threshold, repeatedly until well anastomosed. Another use of DTM is to provide base height for modeling, it will be detailed in section D.

\section{Overlay analysis}

After segmentation examination, some discrete points are still out of building contours. There are many reasons that causes these gross error points. On the one hand, these points might be the reflection points from tall trees. On the other hand, in the histogram thresholding process, the optimal threshold is a close value, a few ground points near threshold are segmented by mistake. Moreover, the elevations of some points on high topography are higher than those on low-lying buildings. We eliminate the points out of building contours by overlapping DLG and point cloud. The plane precision of large scale DLG is usually higher than point cloud data. Comparing with other methods, we can not only greatly reduce the acquisition time of building outlines, but also improve the accuracy of plane position. In essence, the overlapping is a filtering process. In GIS platform, intersection analysis between DLG and point cloud data is able to link points attributes inside building contours with buildings.

\section{Building reconstruction}

After intersection analysis, we obtain the elevation of each buildings by calculating arithmetic mean of points elevation inside building contours. It's worth noting that the elevation of buildings is not the actual height above ground. Because the elevation of airborne LiDAR data is absolute elevation, and the building height is the vertical distance from building top to ground. In order to calculate actual height of buildings, we consider using DTM as datum plane to get base height of each buildings. 
According to building height and planar contour provided by $\mathrm{DLG}$, we acquire the $3 \mathrm{D}$ information of each buildings to reconstruct.

\section{EXPERIMENTAL RESULTS}

Case study is completed in ArcGIS, SPSS, GlobalMap, etc. In data preparation, we get .LAS standard format data by POS and ALS processing first. Point cloud is seted projection coordinate, and outputted to shapefile format vector point. Similarly set the projection coordinate of DLG and output shapefile format polygon. For coordinate transformation, we unify DLG and point cloud to Xi'an 80 coordinate system.

Historgram threshold segmentation is divided into 3 steps: (1) generate elevation-frequency histogram by SPSS. Choose $3 \mathrm{~m}$ as statistics interval and determine the peak. as in Figure 2, it's $11.5 \mathrm{~m}$. (2) Confirm the extremal points, it's $7.5 \mathrm{~m}$ and $16.5 \mathrm{~m}$. (3) Merge intervals, elevation of points $<15.5 \mathrm{~m}$ regard as ground points, the others treat as building points.

Histogram

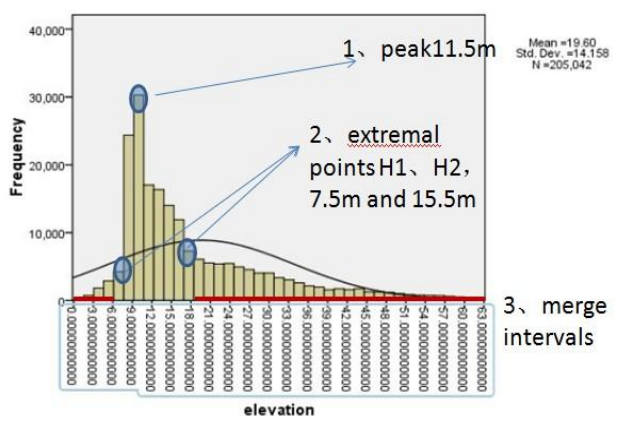

Fig.2. Histogram diagram segmentation

By Kringing interpolation on ground points, we generate DTM to inspect building points. In Figure 3(1), the color depth of DTM presents elevation trend. In Figure 3(2), after superposition of DTM and building points, we can find out there are a number of discrete points in low elevation(light color) area. Figure 3(3) is the chart after elimination. If the test results isn't ideal, there are large number of discrete points(the number of discrete points/the number of total points $>1 \%$ ), we turn back to histogram threshold segmentation until achieve the optimal threshold.

As shown in Figure 4, this article carry on overlapping analysis on building points and DLG after DTM test. (1) is the DLG after vectorization. (2) is the overlaid map of building points and DLG. We can see that some points are out of building contours. (3) is the overlaid map after intersection. All points outside the building contours are eliminated, and polygons' attributes of each buildings correspond to building elevation.

Finally, we calculate the points' mean elevation and add elevation value to attribute table of each building in ArcGIS. In the process of $3 \mathrm{D}$ reconstruction, we choose ArcScene as software platform, importing DLG in which has building elevation property. After selecting DTM as base height, we are able to build 3D models. The effect picture of 3D reconstruction in study area is shown in Figure 5.

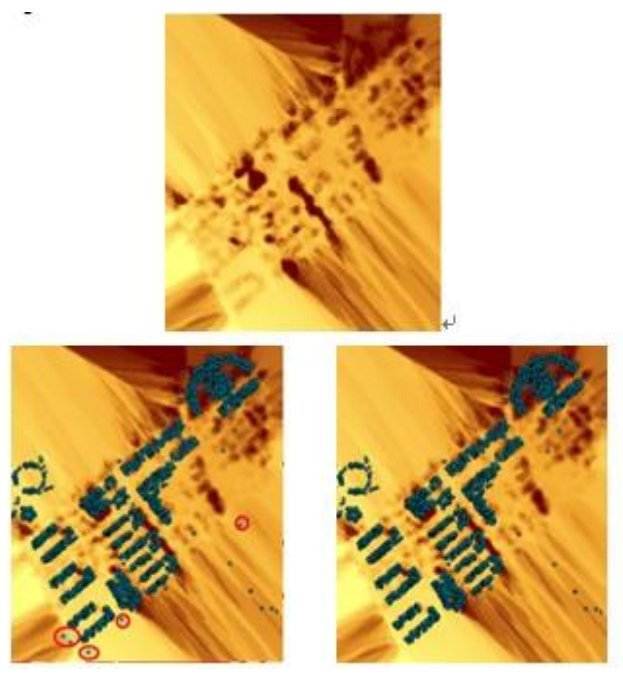

(1) DTM

(2) Superposition of DTM and building points(Inside red circle is the gross error points)

(3) Superposition of DTM and building points after elimination Fig.3. DTM test

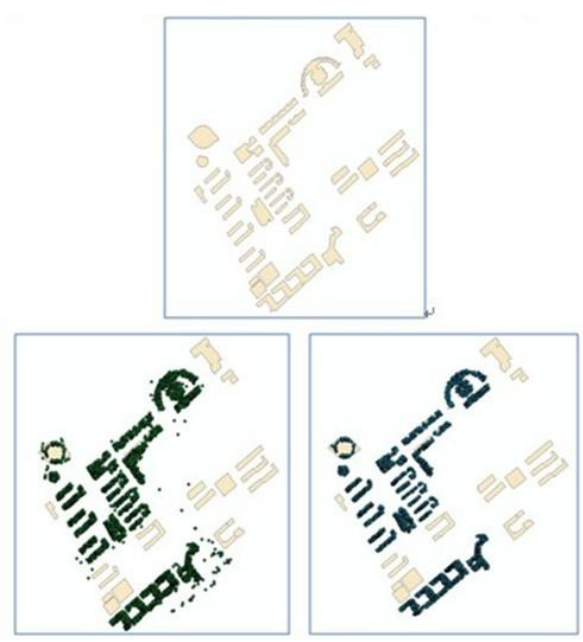

(1) DLG after vectorization

(2) The overlaid map of building points and DLG

(3) The overlaid map after intersection Fig.4. Overlay analysis

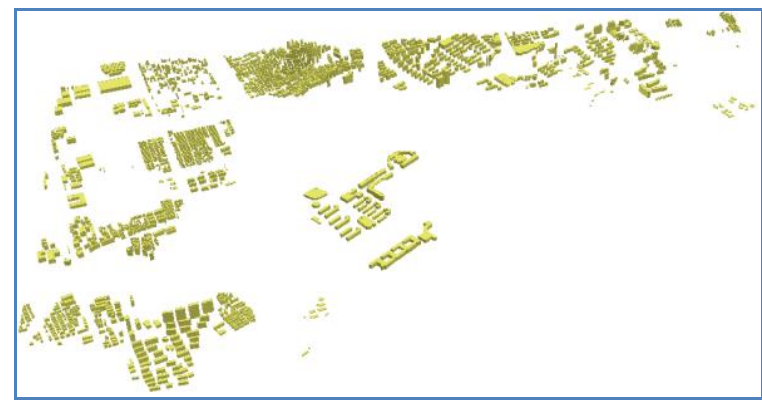

Fig.5. The effect picture of 3D reconstruction 


\section{RESUlT EVALUATION}

The study was conducted in urban district of $17.8 \mathrm{~km}^{2}$. From data processing to modeling accomplishment, it only takes 3 hours. Among them, data processing expenses 1.5 hours, segmentation and test expense 1 hour, modeling takes 0.5 hour.

A prominent feature of this study is low requirement of point cloud density. Nowadays, the point cloud density of mainstream brand airborne LiDAR system can reach 3 to 4 points per square meter. Higher density of point cloud means more cost in data acquisition and slower processing speed. Point cloud density for experiment in this paper is only 0.2 point per square meter. Realization of $3 \mathrm{D}$ reconstruction of buildings with necessary accuracy, is one of important goals in this research. In order to evaluate the suitability of the large area of building reconstruction and to explore modeling effect of different point density, we diluted the original point cloud for 6 times. Each time vacuate about $50 \%$ of the amount of point cloud. The parameters of evalution including point density, completion rate of building reconstruction, modeling time. The experimental results are shown in TABLE I .

Seen from the table, with decreasing density of point cloud, the completion rate and modeling time reduce. After 3 times dilution, completion rate of building reconstruction can reach $91.51 \%$, while the point density is only $0.024 / \mathrm{m}^{2}$, and the modeling time is only $2 \mathrm{~h} 30 \mathrm{~min}$. In former 3 times dilution, the main reason of completion reduction is the vectorization error of DLG. The decrease of modeling principally lies in the reduction of data. The fourth to sixth time dilution, because of the point density is too low and that the area of small buildings is usually less than $100 \mathrm{~m}^{2}$, it began to miss a large number of buildings.

TABLE I. The evaluation of building reconstruction

\begin{tabular}{|l|l|l|l|l|}
\hline $\begin{array}{l}\text { vacuation } \\
\text { frequency }\end{array}$ & $\begin{array}{l}\text { number } \\
\text { of points }\end{array}$ & density & $\begin{array}{l}\text { completion } \\
\text { rate }\end{array}$ & time \\
\hline Original data & 3559068 & 0.198899 & $99.87 \%$ & $3 \mathrm{~h}$ \\
\hline First time & 1779534 & 0.099449 & $99.58 \%$ & $3 \mathrm{~h}$ \\
\hline second time & 889767 & 0.049725 & $97.03 \%$ & $2 \mathrm{~h} 40 \mathrm{~min}$ \\
\hline Third time & 444883 & 0.024862 & $91.51 \%$ & $2 \mathrm{~h} 30 \mathrm{~min}$ \\
\hline Fourth time & 222441 & 0.012431 & $81.46 \%$ & $2 \mathrm{~h} 20 \mathrm{~min}$ \\
\hline Fifth time & 111220 & 0.006216 & $67.53 \%$ & $2 \mathrm{~h} 10 \mathrm{~min}$ \\
\hline Sixth time & 55610 & 0.003108 & $50.38 \%$ & $2 \mathrm{~h}$ \\
\hline
\end{tabular}

\section{CONCLUSION}

Rapid modeling by LiDAR data has been one of the hottest issues in relevant disciplines. The study in this paper is based on GIS, combining with DLG as building contour. It decreases the time of extracting contour from point cloud with necessary accuracy. There is no texture information in building model in this study, and it belongs to white building models. Author thinks there isn't too high requirements on building details for the application such as city planning, land monitoring, land intensive use evaluation etc. Further study will focus on modeling method application in plot ratio and vegetation coverage. In addition, land use classification[9] through point cloud and CCD image will be studied. Above all, the method in this paper is simple and practicable, and it has a broad engineering application prospect.

\section{ACKNOWLEDGMENT}

This research was supported by the National Public Benefit Research Foundation of China (No. 201111014). The authors would like to thank members of Prof.Zheng's group in China University of Geosciences. The authors also thank the reviewers for their valuable suggestions and comments.

\section{REFERENCES}

[1] Charalambos Poullis, Suya You, "Automatic reconstruction of cities from remote sensor data", 2009 IEEE conference on computer vision and pattern recognition,vol. 1-4. pp.2775-2782.

[2] Gianfranco Forlani, Carla Nardinocchi, Marco Scaioni, Primo Zingaretti, "Complete classification of raw LIDAR Data and 3D reconstruction", Pattern analysis and applications, vol. 8. pp. 357-374, Feb 2006.

[3] Ee Hui Lim, David Suter, "3D terrestrial LIDAR classifications with super-voxels and multi-scale conditional random fields", Computer-Aided Design, vol 41. pp.701-710, Oct 2009.

[4] Susanne Becker, "Generation and application of rules for quality dependent facade reconstruction", ISPRS Journal of Photogrammetry and Remote Sensing, vol. 64. pp. 640-653, Nov 2009.

[5] F.Karsli, O.Kahya, "Detecting the buildings from airborne laser scanner data by using Fourier Transform", Experimental Techniques, vol. 36. pp.5-17, Jan-Feb 2012.

[6] Liang-Chien Chen, Tee-Ann Teo, Jiann-Yeou Rau, Jin-King Liu, Wei-Chen Hsu, "Building reconstruction from LIDAR data and aerial imagery", IEEE International Symposium on Geoscience and Remote Sensing, vol. 1-8. pp.2846-2849, 2005.

[7] Yongjun Zhang, Zuxun Zhang, Jianqing Zhang, Jun Wu, "3D building modelling with digital map, LIDAR data and video image sequences", Photogrammetric Record, vol. 20. Pp.285302, Sep 2005.

[8] YOU Hong-jian, ZHANG Shi-qiang, "Reconstructiong 3D buildings based on airborne CCD image and laser scanning rangefinder data", Optics and Precision Engineering, vol. 14. pp.297-320, April 2006.

[9] Yuan Feng, "Research on data processing and classification of land use of airborne LIDAR data", China University of Mining and Technology, 2010. 